\title{
Research of the Leukocytes Segmentation Method in the Blood Cells Recognition Systems
}

\section{Nikitaev V.G., Nagornov O.V., Pronichev A.N., Polyakov E.V., Zaytsev S.M., Zakharenko Y.V., and Dmitrieva V.V.}

National Research Nuclear University MEPhI (Moscow Engineering Physics Institute), Kashirskoe shosse 31, 115409, Moscow, Russia

\section{Annotation}

The paper proposed a method of segmentation of leukocytes from the blood and bone marrow samples. A study of the accuracy characteristics of the method is carried out. The techniques of watersheds and the distances transformation were used as the basis of our segmentation method to achieve optimal segmentation of leukocyte. Proposed method was successfully embedded in the system of automatic recognition of white blood cells for classification of blood cells.

Corresponding Author:

E. Polyakov

EVPolyakov@mephi.ru

Received: 17 January 2018

Accepted: 25 March 2018

Published: 17 April 2018

Publishing services provided by Knowledge

(c) Nikitaev V.G. et al. This article is distributed under the terms of the Creative Commons

Attribution License, which permits unrestricted use and redistribution provided that the original author and source are credited.

Selection and Peer-review under the responsibility of the PhysBioSymp17 Conference Committee.

\section{Introduction}

The use of methods and means of digital image processing for automation of diagnosis of acute leukemia is of considerable interest, because the composition of the cells and their morphological affiliation is of paramount importance [1-2].

The procedure of cells recognition includes the following stages: registration of the images, preprocessing, segmentation, description, classification. One of the most important steps is segmentation, in which nucleus and cytoplasm of cells are selected. Then objects characteristics are measured. Closely located cells on images of bone marrow samples are a challenge for their automatic segmentation [3-4].

A system of blood analysis was presented in [5]. It carries out leukocytes count, leukocytes classification, red blood cells count. The problem of agglomerated leucocytes was solved by distance transformation and watershed algorithm using. Thus, the result is multiple objects, separated by a line roughly coinciding with the line of contact. These objects are an input to the classifier [5]. This method is suitable for separating multiple leukocytes, since the watershed algorithm can detect all areas in 
the transformed image [6]. The disadvantages are the impossibility to apply it to the cells during the adhesion formed ring (the inner line of contact will not be included in the circuit). A remote filter before transformation by the algorithm of the watershed was used in [6]. The algorithm of the watershed with markers was used [6]. This approach is convenient and suitable for the task at hand. It is possible to offer the method proposed in [5] and [6] for image processing in combination of the pros and cons of the considered approaches. Preliminary study of the methods proposed in these papers for the segmentation of cells in bone marrow showed precision in $\sim 55 \%$. So it is necessary to carry out research in the area of modification the proposed methods.

The aim of the paper was to study the effectiveness of methods for distances transformation and watershed in resolving the problem of segmentation of leukocytes in terms of their proximity ("clumping") on the images of bone marrow samples.

\section{Materials and methods}

The process of cells images reception is shown in Figure 1. Images are received by the system of computer microscopy (automated Olympus BX43 microscope with camera Imperx IPX-4M1ST-GCFB). Images were saved in BMP format, color-coded RGB24 ( 16 million colors). 50 samples images were received from which the 1018 cells images were formed $[1-4,7-8]$.

The following steps for image processing are proposed to solve the problem of segmentation - background and erythrocytes removal based on the analysis of luminance histograms of the color component, selecting objects (filling), screening artifacts, the identification of "sticky" white blood cells. The object contours are searched for sticky leukocytes, internal empty space is selected. the distance transform and modified watershed method are used for the separation of sticky white blood cells.

\section{Results and discussion}

The proposed solution is implemented as a software module in C++ using Qt library.

This approach allowed us to effectively separate the sticky cells and isolate their nuclei (Figure 2). The type of the analyzed object influences on the correctness of the algorithm: if the white blood cells stuck together in a closed structure, the division may be wrong. The calculated shape factor makes it easy to distinguish a rounded nucleus from the nucleus of irregular shape that provides valuable information for recognition. 


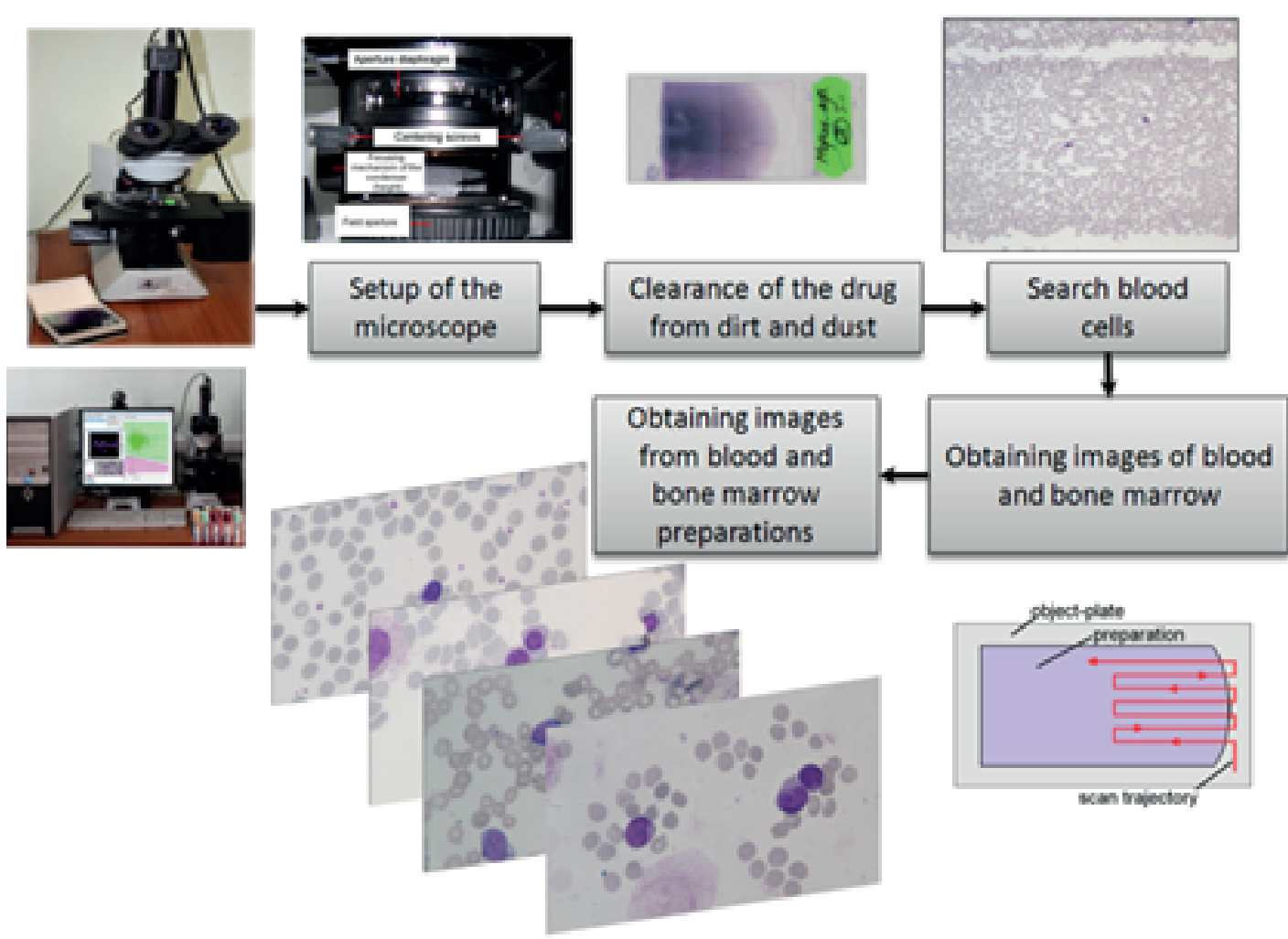

Figure 1: The process of obtaining the images.

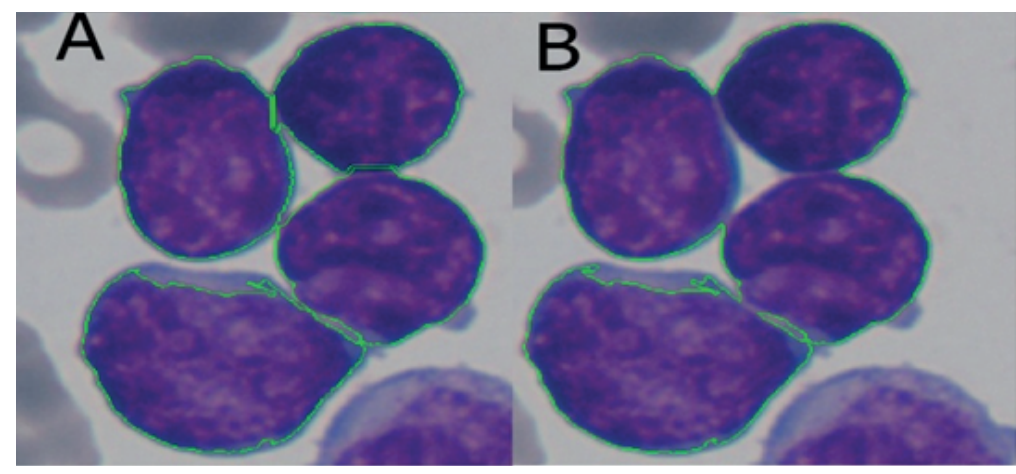

Figure 2: Example of separated (A) and not separated (B) leukocytes.
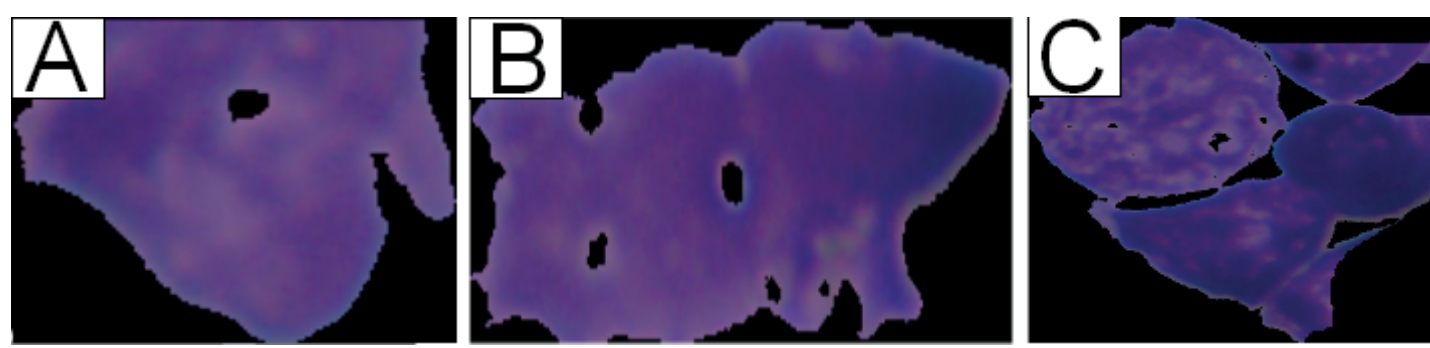

Figure 3: Examples of different types of artifacts: (a) an first type artifact, b) an the second type artifact, c) third type artifact.

Each nucleus of cell from 1018 cells images was assigned to one of the types. 82\% of nucleus were allocated correctly. Were reviewed and categorized the artifacts of 
segmentation. We selected 3 types of artifacts (Figure 3 ). First type artifacts were allocated in $8 \%$ of cells, the second type artifacts were $4 \%$, the third type artifacts were $12 \%$.

\section{Conclusions}

The proposed approach allows leukocytes segmentation in automated recognition of leukocytes.

The segmentation artifacts were identified in this study. We specified three types of artifacts.

We plan to continue research on optimization of the proposed approach with consideration of artifacts at the initial stage of the method.

\section{Acknowledgement}

The reported study was funded by RFBR according to the research project № 17-0701496

\section{References}

[1] V.G. Nikitaev, A.N. Pronichev, E.V. Polyakov, V.V. Dmitrieva, N. N. Tupitsyn, M.A. Frenkel, A.V. Mozhenkova, "Application of texture analysis methods to computer microscopy in the visible range of electromagnetic radiation", Bulletin of the Lebedev Physics Institute, vol. 43, no. 10, pp. 306-308, 2016.

[2] A.N. Pronichev, E.V. Polyakov, N.N. Tupitsyn, M.A. Frenkel, A.V. Mozhenkova, "The use of optical microscope equipped with multispectral detector to distinguish different types of acute lymphoblastic leukemia", Journal of Physics: Conference Series, vol. 784, no. 1, p. 012003, 2017.

[3] Zakharenko Y. V., V. G. Nikitaev, E. V.Polyakov and S. O. Seldyukov "The method of selection of leukocytes in images of preparations of peripheral blood and bone marrow" //Journal of Physics: Conference Series, vol. 798, no. 1, p. 012127, 2017.

[4] A. V. Mozhenkova, N. N. Tupitsin, M. A. Frenkel, N. A. Falaleeva, V. G. Nikitaev and E. V. Polyakov "Computer microscopy in lymphoma diagnostics", Journal of Physics: Conference Series. vol. 798, no. 1, p. 012126, 2017.

[5] L Putzu and C Di Ruberto "White Blood Cells Identification and Counting from Microscopic Blood Image", International Journal of Medical, Health, Biomedical, 
Bioengineering and Pharmaceutical Engineering, vol.7, no.1, Pp. 20-27, 2013

[6] P. P. Acharjya, A. Sinha, S. Sarkar, S. Dey, and S. Ghosh (2013), "A new approach of watershed algorithm using distance transform applied to image segmentation", International Journal of Innovative Research in Computer and Communication Engineering, vol., 1, no. 2, pp. 185-189, 2013

[7] V.G. Nikitaev, O.V. Nagornov, A.N. Pronichev, E.V. Polyakov, V.Y. Sel'chuk, K.S. Chistov, V.N. Blindar', V.V. Dmitrieva, S.M Zaitsev, V.V. Gordeev, "Study of the Effectiveness of Using Wavelet Analysis in Data-Acquisition Systems for Diagnosis of Acute Leukemias" Measurement Techniques, vol. 57, no. 10, pp. 1203-1208, 2015.

[8] V.G. Nikitaev, O.V. Nagornov, A.N. Pronichev, E.V. Polyakov, V.V. Dmitrieva, "The use of the wavelet transform for the formation of the quantitative characteristics of the blood cells images for the automation of hematological diagnostics" WSEAS Transactions on Biology and Biomedicine, vol. 12, no. 3, pp. 16-19, 2015. 